\title{
Preparation of Nanoporous Polymer Films for Real-Time Viability Monitoring of Cells
}

\author{
Chia-Man Chou, ${ }^{1}$ Tong-You Wade Wei, ${ }^{2}$ Jou-May Maureen Chen, ${ }^{2}$ Wei-Ting Chang, ${ }^{3}$ \\ Chang-Tze Ricky Yu, ${ }^{2}$ and Vincent K. S. Hsiao ${ }^{2,4}$ \\ ${ }^{1}$ Division of Pediatric Surgery, Department of Surgery, Taichung Veterans General Hospital, 160 Section 3 Chung-Kang Road, \\ Taichung 40705, Taiwan \\ ${ }^{2}$ Graduate Institute of Biomedicine and Biomedical Technology, National Chi Nan University, 1 University Road Puli, \\ Nantou 54561, Taiwan \\ ${ }^{3}$ Department of Applied Chemistry, National Chi Nan University, 1 University Road Puli, Nantou 54561, Taiwan \\ ${ }^{4}$ Department of Applied Materials and Optoelectronic Engineering, National Chi Nan University, 1 University Road, Puli, \\ Nantou 54561, Taiwan
}

Correspondence should be addressed to Vincent K. S. Hsiao, kshsiao@ncnu.edu.tw

Received 19 May 2010; Accepted 1 July 2010

Academic Editor: Lu Sun

Copyright (C) 2011 Chia-Man Chou et al. This is an open access article distributed under the Creative Commons Attribution License, which permits unrestricted use, distribution, and reproduction in any medium, provided the original work is properly cited.

We have demonstrated an alternative way to monitor the viability of cells adhered on a nanoporous polymer film in real time. The nanoporous polymer films were prepared by laser interference pattering. During exposure of holographic patterning, the dissolved solvents were phase separated with photocured polymer and the nanopores were created as the solvents evaporated. The diffracted spectra from the nanoporous polymer film responded to each activity of the cell cycle, from initial cell seeding, through growth, and eventual cell death. This cell-based biosensor uses a nanoporous polymer film to noninvasively monitor cell viability and may prove useful for biotechnological applications.

\section{Introduction}

Nanoporous materials with a pore size ranging from $1 \mathrm{~nm}$ to $100 \mathrm{~nm}$ have demonstrated applications in a number of multidisciplinary fields [1]. The large internal surface area of nanoporous materials optimizes their use as a functional structure which absorbs or interacts with atoms, ions, and molecules. In biosensing applications [2-7], the porosity yields a large surface-to-volume ratio that enables the immobilization and hybridization of a higher percentage of analyte, such as protein or DNA. The selectivity and sensitivity of nanopore-based biological sensors are dramatically increased by designing nanopores with different surface modification techniques [8-13]. Polymeric materials are widely used in biological applications [14-17] because of the ease of synthesis and intrinsic functional properties, such as biodegradability and biocompatibility, which are key characteristics for cell and tissue engineering. Polymeric materials have also attained much attention in nanoscience and nanotechnology as they can be designed for advanced, smart applications. Several techniques, such as soft lithography, template-directed emulsion, and polymerizationinduced phase separation, have been demonstrated as practical means to fabricate nanoporous polymers with different functionalities [18-23]. We have developed a system to fabricate nanoporous polymers by holographic interference patterning using a photo-curable monomer containing a polar solvent (formamide) or solvent/liquid crystal (LC) mixture $[24,25]$. The nanoporous structure is determined by the interference pattern generated by one or more laser beams. We have also demonstrated that such polymeric, nanoporous photonic structures could be used as sensitive and selective sensors that detect solvent vapor, relative humidity, and protein [26-31].

In this paper, we demonstrate a cell-based biosensor using nanoporous polymeric film. The transmitted spectrum 


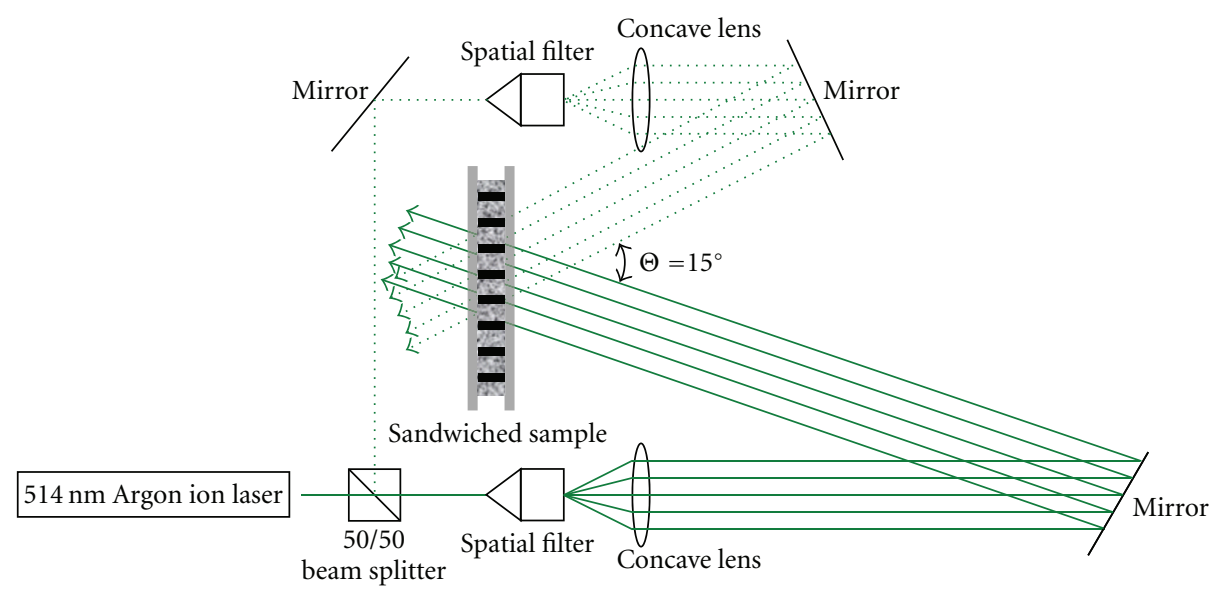

FIgURE 1: The schema of optical setup for fabricating the nanoporous polymer film.

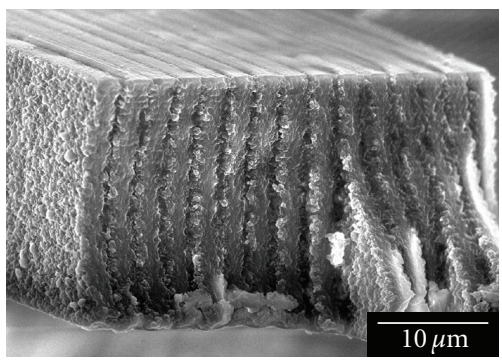

FIGURE 2: SEM cross-sectional micrograph of the nanoporous polymer film.

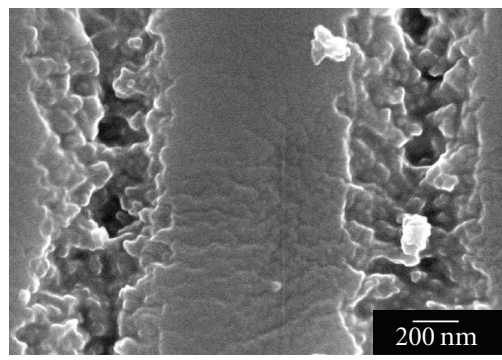

FIGURE 3: SEM surface morphology of nanopores.

from an ordered, nanoporous polymer substrate varies from initial cell seeding, during cell growth, and cell death. This spectal-based analysis provides an alternative way to monitor cell culture viability using optical transducer scheme, which has advantage of label-free detection, in contrast to fluorescence detection. HeLa, a common cervical cancer cell line, served as the cell model for the studies.

\section{Experimental Section}

A photo-curable monomer containing $10 \mathrm{wt} \%$ 3-aminopropyltriethoxysilane (APTES, Aldrich), $20 \mathrm{wt} \%$ acetone solution (Aldrich), $20 \mathrm{wt} \%$ liquid crystal (MDA3061, Merck Taiwan), $40 \mathrm{wt} \%$ dipentaerythritol hydroxypenta acrylate (Aldrich), $1 \mathrm{wt} \%$ Rose Bengal (Spectra Group Limited), $2 \mathrm{wt} \%$ n-phenylglycine (Aldrich), and $7 \mathrm{wt} \%$ n-vinylpyrrolidinone (Aldrich) was used to fabricate the nanoporous polymer structures. We first mixed the monomer syrup homogeneously with a mixer and sonicator. Second, we sandwiched $20 \mu \mathrm{L}$ of the monomer onto one glass slide and covered the syrup with another ITO-coated glass slide. The thickness of the sandwiched sample was controlled by a $15 \mu \mathrm{m}$ plastic spacer. Third, we used a $514 \mathrm{~nm}$ Argon ion laser as the exposure source to conduct the holographic interference patterning process. During the holographic interference pattering, the sandwiched sample was exposed under two laser beams of $100 \mathrm{~mW}$ at a writing angle of 15 degrees for one minute. Figure 1 shows the schematic of the optical setup for fabricating the nanoporous polymer film by holographic interference patterning. Fourth, immediately following the interference patterning, we postcured the sandwiched sample under a white light source for 24 hours. After the postcuring process, the sandwiched sample was separated for cell seeding. The nanoporous polymer remained upon the non-ITO-coated glass slide. The surface and cross-sectional morphologies of the fabricated nanoporous polymer film were characterized by a low voltage scanning electron microscope (LVSEM). The cervical cancer cell line HeLa was cultured with DMEM medium with 5\% fetal bovine serum, $2 \mathrm{mM}$ L-glutamine, and sodium pyruvate. The nanoporous polymeric sample was sterilized with $70 \%$ ethanol, exposed to UV light for 30 minutes, and subsequently placed in a $6-\mathrm{cm}$ petridish. $3 \times 10^{5} \mathrm{HeLa}$ cells were seeded onto the petridish for 8 hours and the cells attached to the nanoporous polymer were photographed with a microscope (Olympus CKX41) bound CCD camera (Olympus DP71). The transmission spectra from the cell-seeded samples were characterized using an Ocean Optics HR4000 spectrometer. 

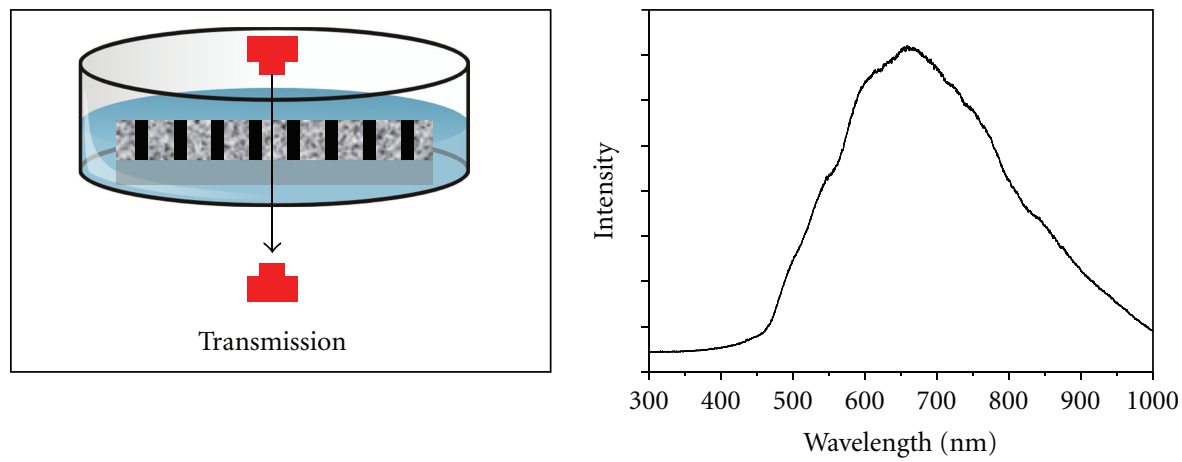

(a)
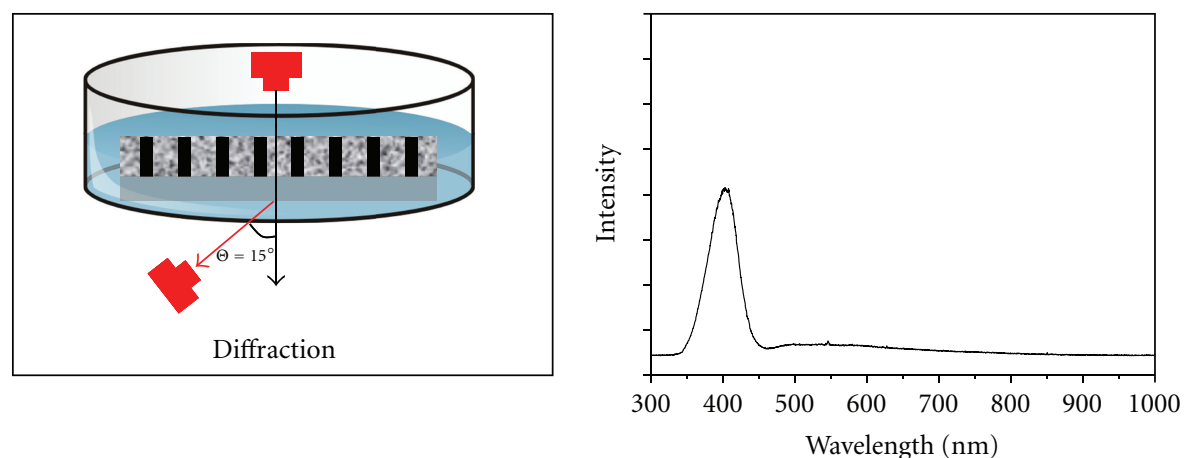

(b)
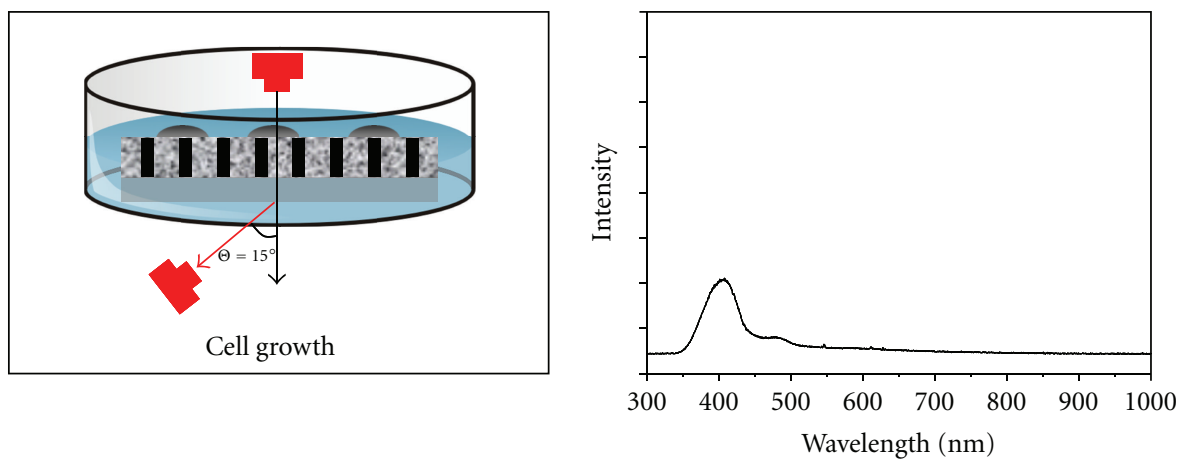

(c)
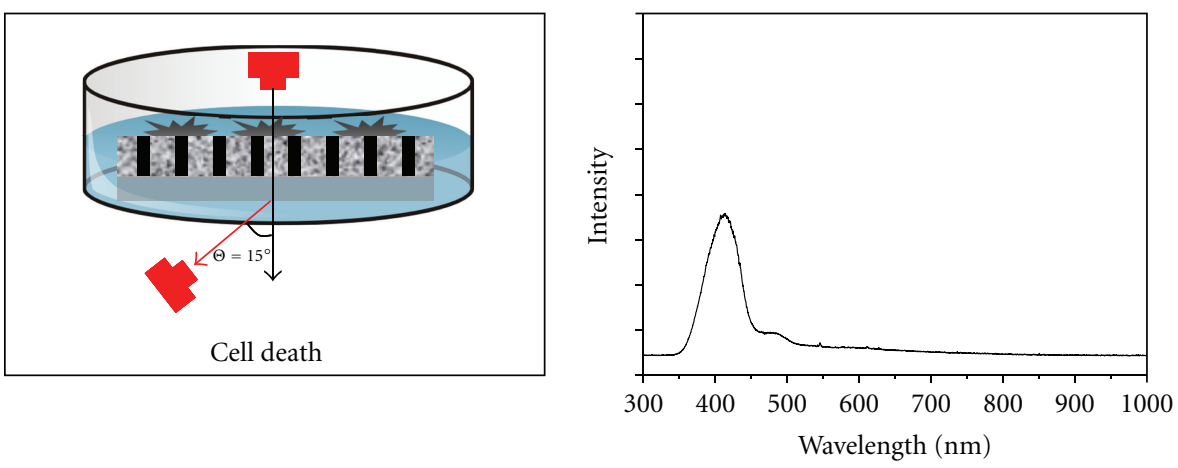

(d)

Figure 4: Optical design used to monitor the biological viablity of the cells using the nanoporous polymer film. On the right are the representative spectra corresponding to the each process. (a) To measure the transmitted light from the sample, illumination (light source) and observation (detector) are aligned on the same optical path and the corresponded spectra shows a broad shape which is not suitable for biological observation. (b) To measure the diffracted light, the observation (detector) is placed in the position of the diffracted light path, $15^{\circ}$ from the sample normal, leading to a sharp spectrum signal. (c) Seeding the cells on the sample surface introduces a scattering effect as the cells scatter some light out of the diffracted light path and the intensity of spectrum decreases. (d) Cell death causes the cells to detach from the sample surface and the intensity of spectrum increases. 


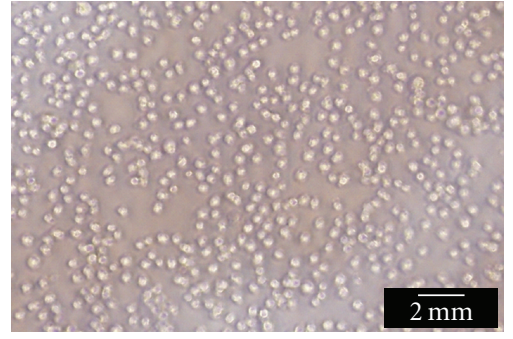

(a)

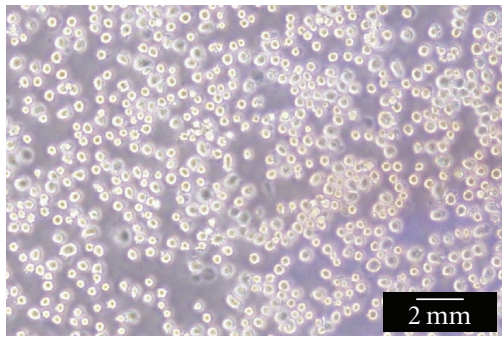

(b)

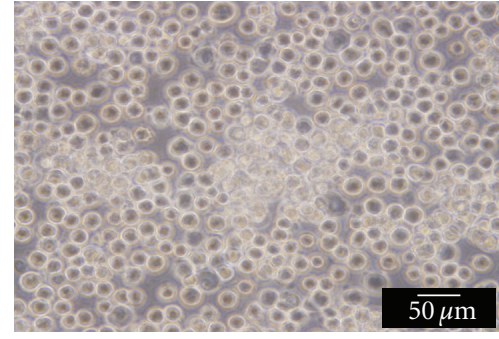

(c)

FIGURE 5: Observation of phase-contrast microscopic images of cervical cancer cells on the surface of nanoporous polymer film (a) before cell seeding; (b) after $8 \mathrm{hrs}$ cell seeding and incubation and (c) after $8 \mathrm{hrs}$ of cell seeding and incubation, and the inducing cell death for $8 \mathrm{hrs}$ at room temperature.

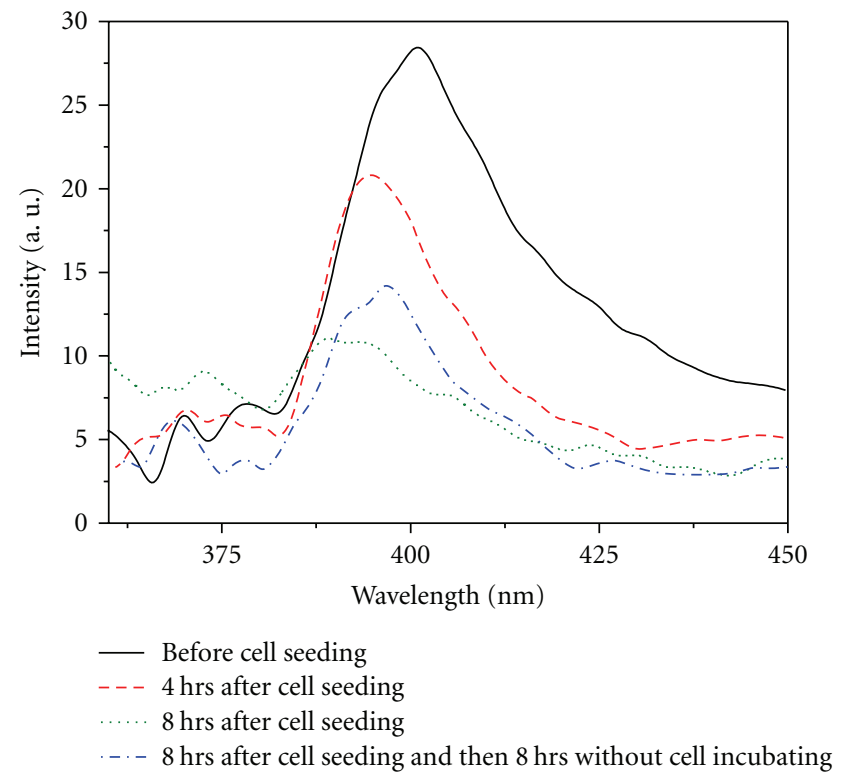

Figure 6: The diffraction spectra characterized from the nanoporous polymer film at different time of cell incubation.

\section{Results and Discussion}

In a typical process of holographic interference patterning using photopolymer and $\mathrm{LC} /$ solvent mixture, the photopolymer is polymerized in the bright region (constructive interference) while the LC/solvent tends to be trapped in the dark region (destructive interference) by the photochemical potential force during the photopolymerization process. Under the photochemically induced phase-separation state, the LC/solvent phase separates from the polymer and generates an ordered structure defined by the holographic interference pattern. After opening the sandwiched sample, the solvent evaporates and a well-defined nanoporous photonic polymer structure is obtained. Figure 2 shows the SEM cross-sectional micrograph of nanoporous polymer film fabricated by two-beam interference patterning. A onedimensional lamella structure consisting of layered polymers and patterned nanopores was successfully prepared by the holographic technique. Figure 3 depicts the surface morphology of the nanopores separated by the polymer. The pore size observed within the polymer lamella ranges between 50 $200 \mu \mathrm{m}$.

Figure 4 shows a schematic illustration of the optical design and corresponding spectra of the cell-based biosensor using the nanoporous polymer film. Since the cell culturing process was conducted in media, the nanoporous polymer film situated on one side of the glass slide has to be stable in the aqueous environment. We have observed that by adding the APTES compound, the nanoporous polymer film's water-stability is strongly enhanced [26]. Moreover, it should be noted that APTES is commonly used for preparing amino functional groups on glass and silica. The APTES-modified materials are biocompatible and suitable for biosensing applications. The addition of APTES in the photopolymer not only enhances the stability, but also the biocompatibility of nanoporous polymer film. When both light source and detector are positioned normal to the sample, the nanoporous polymer film presents a relative spectrum containing a broad peak ranging from $450 \mathrm{~nm}$ to $1000 \mathrm{~nm}$, as shown in Figure 4(a). If the detector is placed at the position of diffracted light, the obtained spectrum shows a sharp peak is observed at the wavelength of $\sim 400 \mathrm{~nm}$, as shown in Figure 4 (b). The introduction of adhered cells on the surface of the nanoporous polymer film generates scattering and the intensity of diffraction decreases, as shown in Figure 4(c). Figure 4(d) depicts the reverse, as the detachment of the cells due to cell death increases the intensity of the light scatter. Herein, lies the sensing mechanism of this biosensor. The light scatter from viable, adhered cells is transduced via the intensity and peakshift properties of the diffraction spectrum.

To verify the cell attachment on the sample surface, optical microscopy was performed on nanoporous polymer during the cell viability. Figures 5(a) and 5(b) show the phase-contrast microscope images for cervical cells seeded on the surface of nanoporous polymer film after $0-8 \mathrm{hr}$ cell incubation, respectively. Cervical cancer cells appear with a small round shape on the surface of nanoporous polymer film. Figure 5(c) shows the image for cervical cells after $8 \mathrm{hr}$ cell seeding and kept in the room temperature without cell incubation. The clear image of cells indicates the cell 
attaching on the surface of nanoporous polymer film while the unclear image (out of focusing) indicates the unattached cells. We speculate that some of the unattached cells are from the dead cells that could be proved from the optical spectroscopy.

The change of light scattering from the biological activity on the nanoporous polymer film was monitored using fiber optical spectroscopy. Figure 6 shows the relative intensity of diffracted light dependent on the time of cell seeding upon the nanoporous polymer film. After $4 \mathrm{hr}$ cell incubating, the diffracted light decreases. Light scattering generated by cell adhesion onto the sample causes a decrease of diffracted light. We also observed that the peak wavelength $(\sim 400 \mathrm{~nm})$ diffracted from the sample blue-shifts $5 \mathrm{~nm}$ after 4 hrs cell growth. According to the Bragg condition, $\lambda=2 n_{\text {ave }} \Lambda$, where $\lambda$ is the peak wavelength of spectrum, $n_{\text {ave }}$ is the average refractive index of sample, and $\Lambda$ is period of the periodic structure, we speculate that the attachment of the cells might induce a change in the refractive index and further change the peak wavelength of the diffracted spectrum. $8 \mathrm{hr}$ cell incubating make the intensity of peak wavelength of spectrum decrease and the wavelength blue-shift $8 \mathrm{~nm}$. We removed the sample from the incubator and recorded the diffraction spectrum after $8 \mathrm{hrs}$ and found that the intensity of the spectrum increased and redshifted $8 \mathrm{~nm}$. The cells should become nonviable upon prolonged periods of time removed from the incubator. The cells detached from the sample and the sample has become more transparent. Such biological activities were recorded by the optical spectrum from the nanoporous polymer film.

\section{Conclusion}

We have successfully prepared a nanoporous polymer film which has served as a platform to observe cell activity. The ordered nanopores were created by a holographic interference pattering technique. The diffracted spectra observed from the nanoporous polymer film responded to the attached cell activity of cell seeding, growth, and death. The cell-based biosensor fabricated by the nanoporous polymer film offers a label-free, noninvasive method to continuously monitor cell activity and may prove useful for biotechnological applications.

\section{Acknowledgments}

This work was supported by Taichung Veterans General Hospital/National Chi Nan University Joint Research Program (TCVGH-NCNU-987907, TCVGH-NCNU-997902). The authors thank John R. Waldeisen for technical discussion and preparation of the paper.

\section{References}

[1] G. Q. Lu and X. S. Zhao, Nanoporous Materials: Science and Engineering, Imperial College Press, London, UK, 2004.

[2] O. A. Saleh and L. L. Sohn, "An artificial nanopore for molecular sensing," Nano Letters, vol. 3, no. 1, pp. 37-38, 2003.
[3] H. Liu, P. He, Z. Li, and J. Li, "High surface area nanoporous platinum: facile fabrication and electrocatalytic activity," Nanotechnology, vol. 17, no. 9, pp. 2167-2173, 2006.

[4] C. R. Martin and Z. S. Siwy, "Learning nature's way: biosensing with synthetic nanopores," Science, vol. 317, no. 5836, pp. 331332, 2007.

[5] S. Benner, R. J. A. Chen, N. A. Wilson et al., "Sequence-specific detection of individual DNA polymerase complexes in real time using a nanopore," Nature Nanotechnology, vol. 2, no. 11, pp. 718-724, 2007.

[6] S. G. Lemay, "Nanopore-based biosensors: the interface between ionics and electronics," ACS Nano, vol. 3, no. 4, pp. 775-779, 2009.

[7] B. M. Venkatesan, B. Dorvel, S. Yemenicioglu, N. Watkins, I. Petrov, and R. Bashir, "Highly sensitive, mechanically stable nanopore sensors for DNA analysis," Advanced Materials, vol. 21, no. 27, pp. 2771-2776, 2009.

[8] H. Chang, F. Kosari, G. Andreadakis, M. A. Alam, G. Vasmatzis, and R. Bashir, "DNA-mediated fluctuations in ionic current through silicon oxide nanopore channels," Nano Letters, vol. 4, no. 8, pp. 1551-1556, 2004.

[9] K. A. Kilian, T. Böcking, K. Gaus, M. Gal, and J. J. Gooding, "Peptide-modified optical filters for detecting protease activity," ACS Nano, vol. 1, no. 4, pp. 355-361, 2007.

[10] Z. Yang, Z. Xie, H. Liu, F. Yan, and H. Ju, "Streptavidinfunctionalized three-dimensional ordered nanoporous silica film for highly efficient chemiluminescent immunosensing," Advanced Functional Materials, vol. 18, no. 24, pp. 3991-3998, 2008.

[11] D. Losic, M. A. Cole, B. Dollmann, K. Vasilev, and H. J. Griesser, "Surface modification of nanoporous alumina membranes by plasma polymerization," Nanotechnology, vol. 19, no. 24, Article ID 245704, 7 pages, 2008.

[12] M. Wanunu, J. Sutin, and A. Meller, "DNA profiling using solid-state nanopores: detection of DNA-binding molecules," Nano Letters, vol. 9, no. 10, pp. 3498-3502, 2009.

[13] V. Mussi, P. Fanzio, L. Repetto et al., "DNA-functionalized solid state nanopore for biosensing," Nanotechnology, vol. 21, no. 14, Article ID 145102, 5 pages, 2010.

[14] T. Cassagneau and F. Caruso, "Conjugated polymer inverse opals for potentiometric biosensing," Advanced Materials, vol. 14, no. 24, pp. 1837-1841, 2002.

[15] J. T. Borenstein, E. Barnard, B. Orrick, W. Cheung, C. Sundback, and J. P. Vacanti, "Microfabricated biodegradable scaffolds for tissue engineering of vital organs," in Proceedings of Materials Research Society Symposium, pp. 9-11, 2004.

[16] X. Wang, Y.-G. Kim, C. Drew, B.-C. Ku, J. Kumar, and L. A. Samuelson, "Electrostatic assembly of conjugated polymer thin layers on electrospun nanofibrous membranes for biosensors," Nano Letters, vol. 4, no. 2, pp. 331-334, 2004.

[17] A. Monge, N. Snejko, E. Gutiérrez-Puebla et al., "One teflon $(\mathrm{B}$-like channelled nanoporous polymer with a chiral and new uninodal 4-connected net: sorption and catalytic properties," Chemical Communications, no. 10, pp. 1291-1293, 2005.

[18] W. Qian, Z.-Z. Gu, A. Fujishima, and O. Sato, "Threedimensionally ordered macroporous polymer materials: an approach for biosensor applications," Langmuir, vol. 18, no. 11, pp. 4526-4529, 2002.

[19] V. Lehmann, "Biosensors: barcoded molecules," Nature Materials, vol. 1, no. 1, pp. 12-13, 2002.

[20] A. Valsesia, P. Colpo, M. M. Silvan, T. Meziani, G. Ceccone, and F. Rossi, "Fabrication of nanostructured polymeric 
surfaces for biosensing devices," Nano Letters, vol. 4, no. 6, pp. 1047-1050, 2004.

[21] S. Sotiropoulou, V. Vamvakaki, and N. A. Chaniotakis, "Stabilization of enzymes in nanoporous materials for biosensor applications," Biosensors and Bioelectronics, vol. 20, no. 8, pp. 1674-1679, 2005.

[22] Q. Li, J. F. Quinn, Y. Wang, and F. Caruso, "Preparation of nanoporous polyelectrolyte multilayer films via nanoparticle templating," Chemistry of Materials, vol. 18, no. 23, pp. 54805485, 2006.

[23] S. Wu, S. R. Park, and X. S. Ling, "Lithography-free formation of nanopores in plastic membranes using laser heating," Nano Letters, vol. 6, no. 11, pp. 2571-2576, 2006.

[24] V. K. S. Hsiao, T.-C. Lin, G. S. He et al., "Optical microfabrication of highly reflective volume Bragg gratings," Applied Physics Letters, vol. 86, no. 13, Article ID 131113, 3 pages, 2005.

[25] V. K. S. Hsiao, K.-T. Yong, A. N. Cartwright et al., "Nanoporous polymeric photonic crystals by emulsion holography," Journal of Materials Chemistry, vol. 19, no. 23, pp. 3998-4003, 2009.

[26] V. K. S. Hsiao, W. D. Kirkey, F. Chen, A. N. Cartwright, P. N. Prasad, and T. J. Bunning, "Organic solvent vapor detection using holographic photopolymer reflection gratings," Advanced Materials, vol. 17, no. 18, pp. 2211-2214, 2005.

[27] X. Fang, V. K. S. Hsiao, V. P. Chodavarapu, A. H. Titus, and A. N. Cartwright, "Colorimetric porous photonic bandgap sensors with integrated CMOS color detectors," IEEE Sensors Journal, vol. 6, no. 3, pp. 661-666, 2006.

[28] V. K. S. Hsiao, J. R. Waldeisen, Y. Zheng, P. F. Lloyd, T. J. Bunning, and T. J. Huang, "Aminopropyltriethoxysilane (APTES)-functionalized nanoporous polymeric gratings: fabrication and application in biosensing," Journal of Materials Chemistry, vol. 17, no. 46, pp. 4896-4901, 2007.

[29] J. Shi, V. K. S. Hsiao, and T. J. Huang, "Nanoporous polymeric transmission gratings for high-speed humidity sensing," Nanotechnology, vol. 18, no. 46, Article ID 465501, 6 pages, 2007.

[30] J. Shi, V. K. S. Hsiao, T. R. Walker, and T. J. Huang, "Humidity sensing based on nanoporous polymeric photonic crystals," Sensors and Actuators B, vol. 129, no. 1, pp. 391-396, 2008.

[31] W. Yan, V. K. S. Hsiao, Y. B. Zheng, Y. M. Shariff, T. Gao, and T. J. Huang, "Towards nanoporous polymer thin film-based drug delivery systems," Thin Solid Films, vol. 517, no. 5, pp. 1794-1798, 2009. 

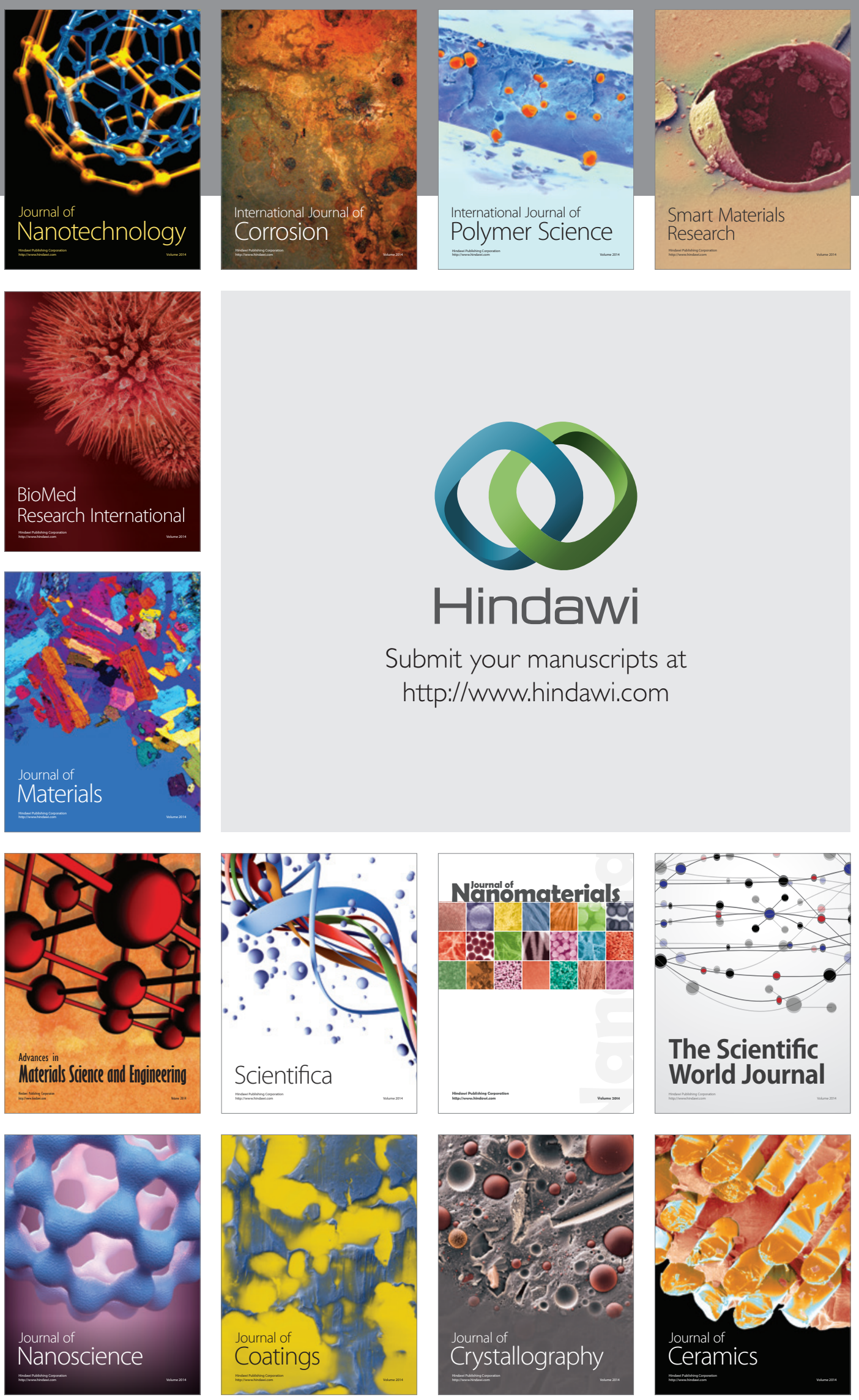

The Scientific World Journal

Submit your manuscripts at

http://www.hindawi.com

\section{World Journal}

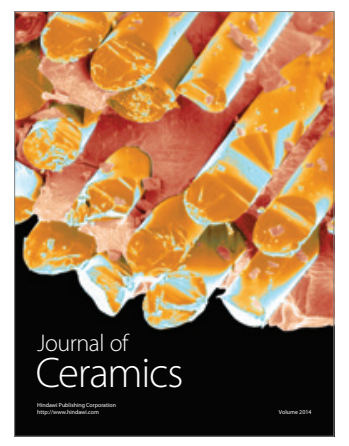

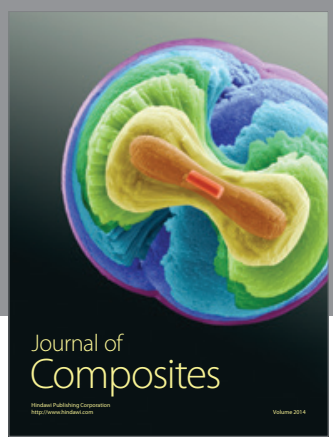
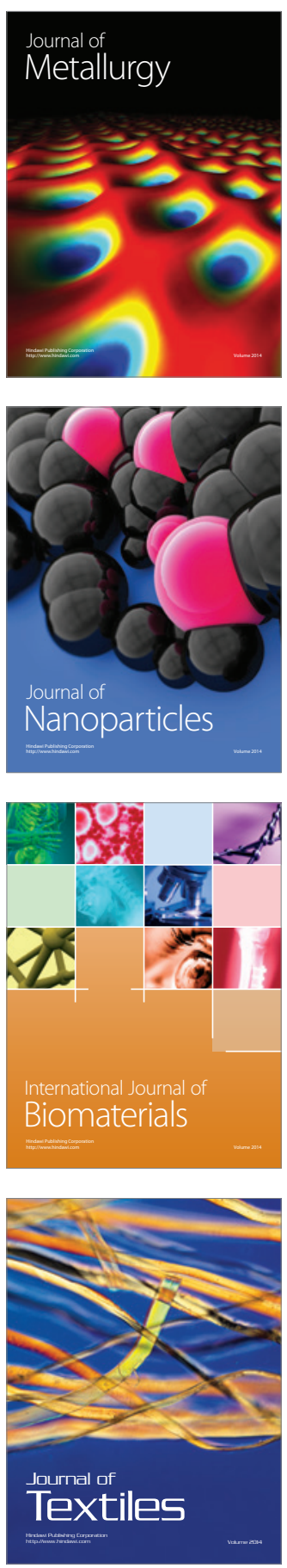